\title{
INTER-RELAÇÃO SEXUAL DE Magnaporthe grisea DO TRIGO E DE OUTROS HOSPEDEIROS
}

\author{
ANA C. BRUNO* \& ALFREDO S. URASHIMA** \\ Departamento de Biotecnologia Vegetal, Centro de Ciências Agrárias, Universidade Federal de São Carlos, Cx. Postal 153, \\ CEP 13600-970 Araras, SP, fax (019) 5423888, e-mail: alfredo@dbv.cca.ufscar.br
}

(Aceito para publicação 02/01/2001)

Autor para correspondência: Alfredo S. Urashima

BRUNO, A.C. \& URASHIMA, A.S. Inter-relação sexual de Magnaporthe grisea do trigo com a brusone de outros hospedeiros. Fitopatologia Brasileira 26:21-26. 2001.

\section{RESUMO}

Foram identificados isolados de Magnaporthe grisea do Brasil a serem utilizados como parentais em estudos de determinação do tipo compatível ("mating type") do fungo. A escolha dos melhores parentais baseou-se naqueles isolados que determinaram o tipo compatível de maior número de isolados de $M$. grisea do trigo (Triticum aestivum). A interrelação sexual da brusone do trigo com a brusone de diversos hospedeiros foi avaliada de acordo com os seguintes parâmetros: determinação de tipo compatível (MAT1-1 e MAT12), sexualidade (hermafrodita, fêmea e macho), fertilidade (número de peritécios) e produção de órgãos sexuais (peritécio, ascas e ascósporos). Os parentais Bp3a e Br118.2D possibili- taram a identificação do tipo compatível de maior porcentagem dos isolados da brusone do trigo. Entre as diversas gramíneas estudadas, os isolados de brusone provenientes de Brachiaria plantaginea e $T$. aestivum podem influenciar na alta variabilidade de $M$. grisea do trigo. Verificou-se variabilidade genética dentro de isolados de Setaria geniculata, sugerindo que a brusone desse hospedeiro pode influir na variabilidade da brusone do trigo. Isolados de brusone do arroz (Oryza sativa) apresentaram baixa fertilidade sexual.

Palavras-chaves: variabilidade, fertilidade, recombinação, tipos compatíveis, ervas daninhas, Triticum aestivum.

\section{ABSTRACT \\ Sexual relationship between Magnaporthe grisea from wheat and from other hosts}

This work aimed to identify the best isolates of Magnaporthe grisea from Brazil to be used as testers for mating type determination of the fungus. The method used was based on the parents that allowed the mating type identification of the greatest number of isolates of M. grisea from wheat (Triticum aestivum). The sexual relationship between the wheat blast fungus and blast strains from other hosts was examined according to the following parameters: determination of mating type (MAT1-1 and MAT1-2), sexuality (hermaphrodite, female, and male), fertility (number of perithecia), and production of sexual organs (perithecia, asci, and ascospores). The testers Bp3a and Br118.2D determined the mating type of $72,7 \%$ of the wheat blast isolates and are recommended for mating type determination. Among the gramineous species, the blast isolates from Brachiaria plantaginea as well as T. aestivum may possibly play a role in the variability of the wheat blast disease. As genetic variability was observed within isolates from Setaria geniculata, blast strain from this weed may also be important in the variability of the wheat blast fungus. The rice (Oryza sativa) blast strain showed low sexual fertility.

\section{INTRODUÇÃO}

Magnaporthe grisea (Hebert) Yaegashi \& Udagawa [anamorfa Pyricularia grisea (Cooke Sacc)] é o patógeno causador da brusone do arroz (Oryza sativa), doença de ocorrência generalizada em todas as regiões rizícolas do mundo. No Brasil, além do arroz, essa doença foi também constatada na cultura do trigo (Triticum aestivum) em 1985,

\footnotetext{
*Bolsista Iniciação Científica FAPESP (98/15702-9)

**Bolsista Pos-Doutor FAPESP (98/03699-3)
}

sendo essa a primeira observação no mundo da doença em condições naturais (Igarashi et al., 1986).

Devido à importância desse cereal no contexto brasileiro e mundial, essa doença tem despertado a atenção de pesquisadores de vários países. Análise da gama de hospedeiros, inoculação cruzada (Prabhu el al., 1992; Urashima et al., 1993; Urashima \& Kato, 1998), fertilidade e compatibilidade sexual (Urashima et al., 1993) aliada ao padrão de bandas de DNA e outros dados moleculares (Lebrun et al., 1990; Valent, 1990; Orbach et al., 1996) demonstraram que os isolados do trigo e do arroz são distintos. 
Na cultura do arroz, onde a pesquisa com brusone vem sendo realizada de longa data e pelos mais diversos centros de pesquisas ao redor do mundo, inúmeras variedades resistentes vem sendo criadas. Mas a vida útil das mesmas tem sido apenas de dois a três anos, pois novas raças do patógeno surgem capazes de quebrar essa resistência. Esse fato tem sido o grande objetivo de todas as pesquisas, ou seja, entender as causas para quebra da resistência dessas variedades em tão curto período. Segundo Zeigler et al. (1995), as explicações para instabilidade da resistência dessas novas variedades podem ser agrupadas em dois grandes grupos: uma seria a exposição inadequada dos materiais à diversidade populacional do patógeno durante os programas de melhoramento, e a segunda a alta variabilidade do fungo causador dessa doença.

Muitos fatores podem contribuir para a geração dessa alta variabilidade. Até recentemente esse fungo era considerado essencialmente assexual, pois a fase sexual somente era obtida em condições de laboratório (Kato, 1977; Yaegashi, 1977; Silué \& Notteghem, 1990). No entanto, recentemente a pesquisa sugere a ocorrência de populações recombinantes num dos centros de origem do arroz (Kumar et al, 1999). A possibilidade da alta variabilidade ser ocasionada pela recombinação sexual é ainda maior para $M$. grisea do trigo, uma vez que o estudo anterior (Urashima et al., 1993), mostrou que os isolados provenientes do trigo produziram peritécios em mais de $93 \%$ dos casos, sendo que em mais de $68 \%$ ocorreu a formação completa dos órgãos sexuais, enquanto que para os isolados do arroz, somente $24 \%$ formaram peritécios, sendo que em nenhum desses ocorreu formação completa dos órgãos sexuais.

Para estudos de compatibilidade sexual de $M$. grisea é necessário inicialmente a determinação do tipo compatível ("mating type") dos isolados. Para isso é necessário a utilização de isolados que já tenham tipo compatível conhecido e que também sejam altamente férteis para serem utilizados como parentais. Estudos de sexualidade realizados até o momento (Yaegashi, 1977; Notteghem \& Silué, 1992; Urashima et al, 1993; Viji \& Gnanamanickam, 1998), utilizaram isolados variados como parentais para esse tipo de análise. Porém, não existe informações quanto a ocorrência de isolados fertéis no Brasil.

O objetivo desse trabalho foi identificar isolados de M. grisea do Brasil que podem ser usados como parentais em estudos de determinação do tipo compatível do fungo.

\section{MATERIAL E MÉTODOS}

\section{Coleção de isolados}

Os isolados de M. grisea utilizados nesse estudo, estavam preservados em sementes autoclavadas de cevada, a temperatura de $5{ }^{\circ} \mathrm{C}$, os quais foram obtidos monosporicamente de lesões típicas. Os isolados utilizados foram coletados de trigo, arroz e ervas daninhas frequientemente encontradas ao redor de lavouras de trigo: Esses foram: Cenchrus echinatus (L.) (2), Echinocloa colonum (Link.) (1), Setaria geniculata (Lam.) Beauv. (3), Digitaria horizontalis Wild. (4), Brachiaria plantaginea (Link.) Hitvhc. (3), T. aestivum (4) e $O$. sativa (3). (Tabela 1 ).

TABELA 1 - Identificação dos isolados de Magnaporthe grisea

\begin{tabular}{ccc}
\hline \hline Isolado & Hospedeiro & Local de origem \\
\hline Br7 & Triticum aestivum & Paraná \\
Br8 & T. aestivum & Paraná \\
Br48 & T. aestivum & Paraná \\
Br118.2D & T. aestivum & Paraná \\
MS05-01 & T. aestivum & Mato Grosso do Sul \\
MS05-03 & T. aestivum & Mato Grosso do Sul \\
MS05-09 & T. aestivum & Mato Grosso do Sul \\
PR02-05 & T. aestivum & Paraná \\
Br35 & Brachiaria plantaginea & Paraná \\
Bp3a & B. plantaginea & São Paulo \\
Br34 & B. plantaginea & Paraná \\
Bp CCA.1 & B. plantaginea & São Paulo \\
Bp CCA. 2 & B. plantaginea & São Paulo \\
VP 05-07 & Oryza sativa & São Paulo \\
VP 07-01 & O. sativa & São Paulo \\
JB 01-11 & O. sativa & São Paulo \\
Sg CCA. 1 & Setaria geniculata & São Paulo \\
Sg CCA. 2 & S. geniculata & São Paulo \\
Br37 & S. geniculata & Paraná \\
Br28 & Digitaria horizontalis & Paraná \\
Br29 & D. horizontalis & São Paulo \\
Br33 & D. horizontalis & Paraná \\
Dh A.R.R.1 & D. horizontalis & São Paulo \\
Br36 & Cenchrus echinatus & Paraná \\
Ce CCA.1 & C. echinatus & São Paulo \\
Br38 & Echinicloa colonum & Paraná \\
\hline &
\end{tabular}

\section{Escolha dos parentais}

A escolha do par de isolados a ser utilizado como parentais foi feita através da capacidade destes em cruzar com maior número de isolados de $M$. grisea. Os isolados foram escolhidos com base na alta fertilidade apresentada em estudos anteriores: Bp3a X Br118.2D (Urashima, 1999), Br7 X Br8 e Br35 X Br48 (Urashima, 1994).

\section{Determinação do tipo compatível ("mating type")}

O método "three-point culture" foi o empregado (Itoi et al., 1983). Fragmentos de micélio dos parentais e de cada um dos isolados de $M$. grisea a serem determinados, foram colocados aproximadamente $4 \mathrm{~cm}$ distantes entre si, em forma de triângulo, em placa de petri com meio de aveia (Avena sativa $\mathrm{L}$.).

Os cruzamentos para determinação dos melhores parentais foram mantidos em sala climatizada a $22{ }^{\circ} \mathrm{C}$, sob bancada asséptica de laboratório e luminosidade constante. Já para os estudos de inter-relação de isolados de brusone de diversos hospedeiros, os cruzamentos foram mantidos em BOD, a $22^{\circ} \mathrm{C}$ sob luz fluorescente contínua. O tipo compatível 
Inter-relação sexual de Magnaporthe grisea do trigo com a brusone de outros hospedeiros

foi determinado após 30 dias entre os isolados compatíveis sexualmente, através da formação de peritécios. Isolados com tipo compatível MAT1-1 foram aqueles que cruzaram com o parental Br118.2D (MAT1-2), e MAT1-2 com o parental Bp3a (MAT1-1).

\section{Sexualidade}

Os cruzamentos entre os isolados férteis das diferentes gramíneas foram classificados quanto à sexualidade em: fêmea, macho (fêmea estéril) e hermafrodita. Isolados fêmea foram aqueles que formaram uma única banda de perítécio no seu próprio lado, machos foram os que formaram uma única banda de peritécio do lado oposto do isolado, ou seja, do lado do parental, e hermafrodita aqueles que formaram duas fileiras de peritécio paralelas na linha de intersecção do crescimento micelial (Itoi et al., 1983).

\section{Fertilidade}

A fertilidade dos isolados de $M$. grisea foi determinada pela capacidade destes de formar peritécio, ascas e ascósporos. Utilizou-se a metodologia adaptada de Viji \& Gnanamanickam (1998). A fertilidade em cada cruzamento foi determinada através do número de peritécios formado em uma área de $25 \mathrm{~mm}^{2}$ sobre a superfície do meio. Os isolados foram classificados em altamente férteis (acima de 20 peritécios $/ 25 \mathrm{~mm}^{2}$ ), intermediária $\left(10\right.$ a 19 peritécios $/ 25 \mathrm{~mm}^{2}$ ), baixa (menos que 10 peritécios $/ 25 \mathrm{~mm}^{2}$ ). Os isolados que não produziram peritécio foram classificados como inférteis.

\section{Produção de órgãos sexuais}

Peritécios maduros foram colocadas em lâminas de vidro, com uma gota de água destilada, e rompidas posteriormente, sob lupa. Em seguida, a presença de ascas e ascósporos foi verificada em microscópio.

\section{RESULTADOS E DISCUSSÃO}

Seis isolados férteis de $M$. grisea foram utilizados a fim de se determinar sua eficiência como parentais para determinação de tipo compatível de isolados de brusone provenientes de cinco lavouras distintas de trigo, distribuídos entre os estados do Mato Grosso do Sul (MS) e Paraná (PR) (Tabela 2). Estes isolados parentais possibilitaram a identificação das características sexuais de 11 isolados de $M$. grisea do trigo, o que correspondeu a 78,5\% dos isolados utilizados, sendo que desse total $72,7 \%$ foi identificado através dos isolados Bp3a x Br118.2D, 18,1\% com os isolados Br35 x Br48 e $18,1 \%$ com $\mathrm{Br} 7$ x Br8. Esses números mostraram a eficiência dos isolados Bp3a e Br118.2D na determinação do tipo compatível de diferentes isolados de $M$. grisea do trigo em relação aos demais parentais. Apesar disso, três isolados somente tiveram seu tipo compatível determinado através dos demais parentais, $\mathrm{Br} 35$ x Br48 e Br7 x Br8. O isolado MS0615 teve seu tipo compatível determinado através de dois pares de parentais, $\mathrm{Br} 7$ x $\mathrm{Br} 8$ e $\mathrm{Br} 35$ x Br48. Interessante observar que o melhor parental Bp3a x Br118.2D é o cruzamento de isolado de $M$. grisea proveniente de dois hospedeiros distintos, B. plantaginea e T. aestivum (Tabela 1), o que demonstra a grande similaridade entre a brusone dessas duas espécies.

Em trabalho anterior Urashima et al. (1993) conseguiram identificar o tipo compatível em mais de $93 \%$ dos isolados. No entanto, nesse estudo os parentais, foram isolados de M. grisea de Eleusine coracona (L.) Gaertn., G101 e Z2-1, que segundo Kato (1977) e Yaegashi (1977), devem ser os recomendados para os estudos de determinação de tipo compatível, por serem os mais férteis. Entretanto, estes isolados não podem ser utilizados em estudos no Brasil, devido à impossibilidade de importação desses fungos para o país. Desse modo, sugere-se a utilização dos parentais Bp3a e Br118.2D como substitutos aos isolados G10-1 e Z2-1 para os estudos de determinação do tipo compatível de $M$. grisea no Brasil.

Dentre todos os isolados testados, apenas três não tiveram o tipo compatível determinado, o que correspondeu a 21,4\%. Um desses isolados, MS06-22 provém do mesmo campo MS06 de outros quatro isolados que tiveram seu tipo compatível facilmente determinado. Isso mostra que mesmo dentro de uma ínfima amostragem ocorreu isolados com diferente fertilidade sexual, confirmando a alta variabilidade genética do fungo.

Em relação à freqüência de cada tipo compatível nas populações dos isolados do PR e MS, notou-se um maior número de isolados com tipo compatível $M A T 1-1$ em relação ao MAT1-2. Dentre os isolados do PR ocorreu somente isolados com tipo compatível MAT1-1, ao passo que no MS, ocorreu tanto MAT1-1 como MAT1-2. A alta incidência de isolados de $M$. grisea do trigo com tipo compatível MAT1-1, também foi verificada por Urashima et al. (1993), que dentre os 16 isolados testados, apenas um apresentou MAT1-2, e um não identificado. Esses dados sugerem que entre os isolados de brusone de trigo, ocorre maior freqüência de apenas um tipo compatível, MAT1-1. A maior frequiência de isolados MAT1-1 também foi observada em M. grisea do arroz por Kato \& Yamaguchi (1982), Yaegashi \& Yamada (1986), Notteghem \& Silué (1992). Embora essa relação desigual na distribuição do MAT1-1 e MAT1-2 tenha sido utilizada no passado como evidência da não ocorrência da reprodução sexual na natureza em populações de $M$. grisea (Nottheghem \& Silué, 1992; Zeigler, 1998), estudos mais recentes, observaram isolados de $M$. grisea do arroz sexualmente férteis na Índia (Kumar et al., 1999) e na Tailândia (Mekwatanakarn et al., 1999) onde inclusive isolados resultantes de recombinação sexual foram detectados (Kumar et al., 1999).

De um campo comercial de trigo do MS foram identificados isolados de $M$. grisea compatíveis (MAT1-1 e MAT12). Esses isolados apresentaram característica de hermafrodita, os quais em condições adequadas de laboratório cruzaram sexualmente, produzindo peritécio, ascas e ascósporos (dados não mostrados). Embora trabalho anterior (Urashima et al., 1993), tenha mostrado a ocorrência de isolados compatíveis em uma mesma região, esta é a primeira evidência da 
importância da reprodução sexual entre isolados compatíveis de uma mesma lavoura, porque produção completa dos órgãos sexuais foram obtidos. Estes dados sugerem que em condições específicas pode ocorrer cruzamento sexual na natureza, com efeitos diretos na alta variabilidade do fungo.

Em relação à sexualidade, verificou-se que $M$. grisea do trigo apresentou alta porcentagem de isolados com característica de fêmea e hermafrodita $(81,8 \%)$ sendo que apenas dois isolados mostraram característica de macho (fêmea estéril) (Tabela 2). Essa característica difere dos isolados de M. grisea do arroz que são quase todos fêmeas estéreis (Kato \& Yamaguchi, 1982; Itoi et al., 1983; Yaegashi \& Yamada., 1986; Notteghem \& Silué, 1992), e os aproxima dos isolados de milhetos e ervas daninhas (Notteghem \& Silué, 1992). Hermafroditismo e feminilidade são pré-requisitos importantes para que haja alta fertilidade e cruzamento sexual entre diferentes isolados de M. grisea.

Quanto à fertilidade sexual dos isolados da brusone do trigo, observou-se incidência de isolados altamente férteis, com produção abundante de peritécio. Esta alta fertilidade foi notada em praticamente todos os isolados do MS, exceto no isolado MS05-01. Os isolados do PR apresentaram diferentes graus de fertilidade, confirmando que ocorre variabilidade entre isolados de $M$. grisea dentro de uma mesma região geográfica.

O presente trabalho também analisou o tipo compatível de $M$. grisea de diferentes hospedeiros (Tabela 3). Todos os isolados foram identificados como MAT1-1, mesmo sendo obtidos de diferentes hospedeiros, pois cruzaram com o isolado Br118.2D (MAT1-2) proveniente de trigo. Esse fato possibilitou analisar a inter-relação sexual de $M$. grisea do trigo com a brusone de outras gramíneas. Essa inter-relação foi analisada de acordo com os seguintes parâmetros: tipo compatível, fertilidade, sexualidade e produção de órgãos sexuais.

Todos os isolados de $D$. horizontalis, mostraram ser MAT1-1, baixa fertilidade, produção de peritécio inferior a 30 em uma área de $25 \mathrm{~mm}^{2}$ na região de confluência, sexua- lidade de macho (fêmea estéril) e produção somente de peritécio, sem ascas e ascósporos. Valent et al. (1986) observaram a relação direta entre macho (fêmea estéril), baixa fertilidade e ausência ou produção incompleta dos órgãos sexuais, o que também foi observada em nosso trabalho. Além disso, a formação somente de peritécio, reforça as observações de Tharreau et al. (1997), de que diferentes genes estão envolvidos na formação de peritécio, ascas e ascósporos. O fato desses isolados terem sido coletados em diferentes regiões de São Paulo e Paraná (Tabela1) mostrou que essa gramínea possuiu uma homogeneidade genética quanto a seus parâmetros sexuais, independentemente do seu local de origem. A não formação de ascósporos, que é a principal estrutura reprodutiva da recombinação sexual, sugere que essa erva daninha não tem qualquer influência na variabilidade da brusone do trigo

Dentre os três isolados de $S$. geniculata coletados de regiões distintas, apenas um $(\mathrm{Br} 37)$ foi compatível com um dos parentais (Br118.2D do trigo), sendo seu tipo compatível MAT1-1, sexualidade de macho e alta fertilidade. Esses dados não estão de acordo com as observações de Valent et al.(1986), pois apesar da sexualidade de macho (fêmea estéril), ocorreu alta formação de peritécio, sem ascas e ascósporos. Em estudos de relação patogênica, esse isolado foi o mais similar à $M$. grisea do trigo, pois foi possível a inoculação cruzada (Urashima \& Kato, 1998). Entretanto, a análise molecular (Urashima et al., 1999) comprovou que esse isolado não apresentou nenhuma similaridade com os isolados do trigo. No entanto, o fato de isolados de $S$. geniculata mostrarem diversidade genética indica que uma procura mais minuciosa de brusone dessa erva daninha se faz necessário, visto que pode ocorrer isolados semelhantes a brusone do trigo.

M. grisea de B. plantaginea também apresentou diversidade genética, uma vez que dos três isolados estudados somente um teve seu tipo compatível identificado (MAT1-1), com produção mediana de peritécio, sexualidade hermafrodita e, conforme observado em estudo anterior (Urashima et al.,

TABELA 2 - Eficiência de três pares de isolados de Magnaporthe grisea para identificação dos parâmetros sexuais

\begin{tabular}{|c|c|c|c|c|c|c|c|c|c|c|c|c|c|c|c|}
\hline \multirow{2}{*}{ Isolado } & \multicolumn{4}{|c|}{ Bp3a x Br118.2D } & \multicolumn{4}{|c|}{ Br35 x Br48 } & \multicolumn{4}{|c|}{ Br7 x Br8 } & \multirow{2}{*}{ Fertilidade } & \multirow{2}{*}{ Sexualidade } & \multirow{2}{*}{$\begin{array}{l}\text { Tipo compatível } \\
\text { ("'mating type") }\end{array}$} \\
\hline & $1 *$ & 2 & 3 & 4 & $1 *$ & 2 & 3 & 4 & $1 *$ & 2 & 3 & 4 & & & \\
\hline PR01-27 & & $\mathrm{X}$ & & & & & & & & & & & $1 * *$ & Fêmea & $M A T 1-1$ \\
\hline \multicolumn{16}{|l|}{ PR01-37 } \\
\hline PR02-05 & & & $X$ & $X$ & & & & & & & & & 3 & Hermafrodita & $M A T 1-1$ \\
\hline PR02-08 & & $\mathrm{X}$ & & $\mathrm{X}$ & & & & & & & & & 2 & Hermafrodita & $M A T 1-1$ \\
\hline PR04-07 & & $X$ & & & & & & & & & & & 1 & Macho & $M A T 1-1$ \\
\hline \multicolumn{16}{|l|}{ PR04-10 } \\
\hline MS05-01 & & $X$ & & & & & & & & & & & 1 & Hermafrodita & $M A T 1-1$ \\
\hline MS05-03 & & $X$ & & & & & & & & & & & 3 & Hermafrodita & $M A T 1-1$ \\
\hline MS05-09 & & & & & & & & & & $\mathrm{X}$ & $\mathrm{X}$ & $X$ & 3 & Fêmea & $M A T 1-2$ \\
\hline MSO6-03 & & $X$ & $X$ & $\mathrm{X}$ & & & & & & & & & 3 & Fêmea & $M A T 1-2$ \\
\hline MS06-13 & & & & & & & $X$ & $X$ & & & & & 3 & Fêmea & $M A T 1-1$ \\
\hline MS06-15 & & & & & & & $\mathrm{X}$ & $X$ & $\mathrm{X}$ & & $\mathrm{X}$ & $\mathrm{X}$ & 3 & Fêmea & $M A T 1-1$ \\
\hline \multicolumn{16}{|l|}{ MS06-22 } \\
\hline MS06-23 & & & $X$ & $X$ & & & & & & & & & 3 & Macho & $M A T 1-1$ \\
\hline
\end{tabular}

* Repetições

**Nota $1=1$ a 5 peritécios ao longo da linha de cruzamento; nota $2=6$ a 15 peritécios e nota $3=16$ a 30 peritécios. 
Inter-relação sexual de Magnaporthe grisea do trigo com a brusone de outros hospedeiros

TABELA 3 - Tipo compatível ("mating-type"), fertilidade, sexualidade e produção de órgãos sexuais de isolados de Magnaporthe grisea de diversos hospedeiros

\begin{tabular}{|c|c|c|c|c|c|c|}
\hline Isolado & Hospedeiro & Tester's & $\begin{array}{c}\text { Tipo } \\
\text { compatível }\end{array}$ & Fertilidade* & Sexualidade & $\begin{array}{c}\text { Órgãos } \\
\text { produzidos }\end{array}$ \\
\hline Ce CCA.1 & Cenchrus echinatus & & N.D.** & & & \\
\hline $\mathrm{Br} 36$ & C. echinatus & & N.D. & & & \\
\hline $\mathrm{Br} 38$ & Echinocloa colonum & & N.D. & & & \\
\hline Sg CCA.1 & Setaria geniculata & & N.D. & & & \\
\hline Sg CCA.2 & S. geniculata & & N.D. & & & \\
\hline $\mathrm{Br} 37$ & S. geniculata & $\mathrm{Br} 118.2 \mathrm{D}$ & MAT 1-1 & Alta & Macho & Peritécio \\
\hline $\operatorname{Br} 29$ & Digitaria horizontalis & $\mathrm{Br} 118.2 \mathrm{D}$ & MAT 1-1 & Baixa & Macho & Peritécio \\
\hline $\operatorname{Br} 33$ & D. horizontalis & $\mathrm{Br} 118.2 \mathrm{D}$ & MAT 1-1 & Baixa & Macho & Peritécio \\
\hline $\operatorname{Br} 28$ & D. horizontalis & $\mathrm{Br} 118.2 \mathrm{D}$ & MAT 1-1 & Baixa & Macho & Peritécio \\
\hline DhARR1 & D. horizontalis & $\mathrm{Br} 118.2 \mathrm{D}$ & MAT 1-1 & Baixa & Macho & Peritécio \\
\hline $\mathrm{Br} 34$ & Brachiaria plantaginea & $\mathrm{Br} 118.2 \mathrm{D}$ & MAT 1-1 & Mediana & Hermafrodita & $\mathrm{P}, \mathrm{A}, \mathrm{As} * *$ \\
\hline Bp CCA.1 & B. plantaginea & & N.D. & & & \\
\hline Вp ССА. 2 & B. plantaginea & & N.D. & & & \\
\hline MS 05-01 & Triticum aestivum & $\mathrm{Br} 118.2 \mathrm{D}$ & MAT 1-1 & Baixa & Hermafrodita & Peritécio \\
\hline MS 05-03 & T. aestivum & $\mathrm{Br} 118.2 \mathrm{D}$ & MAT 1-1 & Baixa & Hermafrodita & Peritécio \\
\hline MS 05-09 & T. aestivum & Вp3a & MAT1-2 & Mediana & Hermafrodita & $\mathrm{P}, \mathrm{A}, \mathrm{As}$ \\
\hline PR 02-05 & T. aestivum & $\mathrm{Br} 118.2 \mathrm{D}$ & MAT 1-1 & Mediana & Hermafrodita & Peritécio \\
\hline JB 01-11 & Oryza sativa & & N.D. & & & \\
\hline VP 07-01 & O. sativa & & N.D. & & & \\
\hline Vp 05-07 & O. sativa & & N.D. & & & \\
\hline
\end{tabular}

*Número de peritécios em $25 \mathrm{~mm}^{2}: 1$-30 Baixa, 30-60 Mediana, >60 Alta.

**N.D. = não determinado; $\mathrm{P}=$ peritécio, $\mathrm{A}=$ Ascas; $\mathrm{As}=$ ascósporos

1993), produção completa dos órgãos sexuais. Esse isolado (Br34) apresentou característica hermafrodita, com formação de duas bandas de peritécio na região de confluência do cruzamento entre os isolados. Segundo Itoi et al. (1983), essa característica está associada aos cruzamentos mais férteis. Nesse sentido, a formação completa dos órgãos sexuais observada no presente trabalho, reforça a relação direta entre sexualidade e produção de órgãos sexuais. $\mathrm{O}$ fato dessas características ter ocorrido entre isolados de $B$. plantaginea e T. aestivum, sugere que esta gramínea comumente encontrada em lavouras de trigo pode influenciar a alta variabilidade do fungo.

Nenhum dos isolados da brusone do arroz provenientes de diferentes regiões, sob diferentes sistemas de cultivo, tiveram seu tipo compatível determinado. Apesar da alta eficiência dos parentais na determinação do tipo compatível de diferentes hospedeiros, no caso do arroz isso não foi possível, comprovando que os isolados de M. grisea desta gramínea possuem baixa fertilidade sexual. Essa característica da brusone do arroz também já foi observada em estudos anteriores (Silué \& Notteghem, 1990; Zeigler, 1998), demonstrando que mesmo com o cultivo de arroz próximo ao trigo, a brusone do arroz não possui nenhum efeito na variabilidade da brusone do trigo.

\section{AGRADECIMENTOS}

Os autores agradecem Y. R. Mehta, M. A. R. Oliveira,
P. G. Sousa, M. T. Fujino, A. G. Linhares e B. A. Welter pelas sementes de trigo.

\section{REFERÊNCIAS BIBLIOGRÁFICAS}

IGARASHI, S., UTIAMADA, C.M., IGARASHI, L.C., KAZUMA, A.H. \& LOPES, R.S. Pyricularia em trigo. 1. Ocorrência de Pyricularia sp. no estado do Paraná. Fitopatologia Brasileira 11:351-352. 1986.

ITOI, S., MISHIMA, T., ARASE, S. \& NOZU, M. Mating behavior of Japanese isolates of Pyricularia oryzae. Phytopatologhy 73:155-158. 1983.

KATO, H. The perfect state of Pyricularia species. Plant Protection 10:20-28. 1977.

KATO, H. \& YAMAGUCHI, T. The perfect state of Pyricularia oryzae Cav. from rice plants in culture. Phytopathology 48:607-612. 1982.

KUMAR, J., NELSON, R.J., ZEIGLER, R.S. Population structure and dynamics of Magnaporthe grisea in the Indian Himalayas. Genetics 152:971-984. 1999.

LEBRUN, M.H., CAPY, M.P., GARCIA, N., DUTERTRE, M., BRYGOO, Y., NOTTEGHEM, J.L. \& VALES, M. Biology and genetics of Pyricularia oryzae and Pyricularia grisea populations: current situation and development of RFLP markets. In: Rice Genetics Conference. Los Baños, IRRI. 1990. Pages: 1-12

MEKWATANAKARN, P., KOSITRATANA, W., PHROMRAKSA, T. \& ZEIGLER, R.S. Sexually fertile 
Magnaporthe grisea rice pathogens in Thailand. Plant Disease 83:939-943. 1999.

NOTTEGHEM, J.L. \& SILUÉ, D. Distribution of the mating type alleles in Magnaporthe grisea populations pathogenic on rice. Phytophatology 82:421-424. 1992.

ORBACH, M.J., CHUMLEY, F.G. \& VALENT, B. Electrophoretic karyotypes of Magnaporthe grisea pathogens of diverse grasses. Molecular Plant-Microbe Interactions 9:261-271. 1996.

PRABHU, A.S., FILIPPI, M.C. \& CASTRO, N. Pathogenic variation among isolates of Pyricularia oryzae infecting rice, wheat, and grasses in Brazil. Tropical Pest Management 38:367-371. 1992.

SILUÉ, D. \& NOTTEGHEM, J.L. Production of perithecia of Magnaporthe grisea on rice plants. Mycological Research 94:1151-1152. 1990.

THARREAU, D., NOTTEGHEM, J.L. \& LEBRUN, M.H. Mutations affecting perithecium development and sporulation in Magnaporthe grisea. Fungal Genetics and Biology 21:206-213. 1997.

URASHIMA, A S. Etiological studies on wheat blast disease caused by Magnaporthe grisea. (Ph.D. Thesis). Kobe. University of Kobe. 1994.

URASHIMA, A.S. Genetic analyses of pathogenicity of Magnaporthe grisea on wheat. Fitopatologia Brasileira 24:567-569. 1999.

URASHIMA, A.S. \& KATO, H. Pathogenic relationship between isolates of Pyricularia grisea of wheat and others hosts at different host developmental stages. Fitopatologia Brasileira 23:30-35. 1998.

URASHIMA, A.S., HASHIMOTO, Y., DON, L.D., KUSABA, M., TOSA, Y., NAKAYASHIKI, H. \&
MAYAMA, S. Molecular analysis of the wheat blast population in Brazil with a homologue of retrotransposon MGR583. Annals of the Phytopathological Society of Japan 65:429-436. 1999.

URASHIMA, A.S., IGARASHI, S. \& KATO, H. Host range, mating type and fertility of Pyricularia grisea from wheat in Brazil. Plant Disease 77:1211-1216. 1993.

VALENT, B. Rice blast as a model system for plant pathology. Phytopathology 80:33-36. 1990.

VALENT, B., CRAWFORD, M.S., WEAVER, C.G. \& CHUMLEY, F.G. Genetic studies of fertility and pathogenicity in Magnaporthe grisea (Pyricularia oryzae). Iowa State Journal Research 60:559-594. 1986.

VIJI, G. \& GNANAMANICKAM, S.S. Mating type distribution and fertility status of Magnaporthe grisea populations from various hosts in India. Plant Disease 82:36-40. 1998.

ZEIGLER, R.S. Recombination in Magnaporthe grisea. Annual Review of Phytopathology 36:249-275. 1998.

ZEIGLER, R.S., CUC, L.X., SCOTT, R.P., BERNARDO, M.A., CHEN, D.H., VALENT, B. \& NELSON, R. The relationship between lineage and virulence in Pyricularia grisea in the Philippines. Phytopathology 85:443-451. 1995.

YAEGASHI, H. On the sexuality of blast fungi, Pyricularia spp. Annals of the Phytopathological Society of Japan 43:431-439. 1977.

YAEGASHI, H. \& YAMADA, M. Pathogenic race and mating type of Pyricularia grisea from Soviet Union, China, Nepal, Thailand, Indonésia and Colombia. Annals of the Phytopathological Society of Japan 52:225-234. 1986. 